\title{
Homotopy Analysis Method for Second-Order Boundary Value Problems of Integrodifferential Equations
}

\author{
Ahmad El-Ajou, ${ }^{1}$ Omar Abu Arqub, ${ }^{1}$ and Shaher Momani ${ }^{2}$ \\ ${ }^{1}$ Department of Mathematics, Faculty of Science, Al Balqa Applied University, Salt 19117, Jordan \\ ${ }^{2}$ Department of Mathematics, Faculty of Science, University of Jordan, Amman 11942, Jordan
}

Correspondence should be addressed to Omar Abu Arqub, o.abuarqub@bau.edu.jo

Received 1 April 2012; Accepted 19 June 2012

Academic Editor: Gabriele Bonanno

Copyright (C) 2012 Ahmad El-Ajou et al. This is an open access article distributed under the Creative Commons Attribution License, which permits unrestricted use, distribution, and reproduction in any medium, provided the original work is properly cited.

\begin{abstract}
In this paper, series solution of second-order integrodifferential equations with boundary conditions of the Fredholm and Volterra types by means of the homotopy analysis method is considered. The new approach provides the solution in the form of a rapidly convergent series with easily computable components using symbolic computation software. The homotopy analysis method provides us with a simple way to adjust and control the convergence region of the infinite series solution by introducing an auxiliary parameter. The proposed technique is applied to a few test examples to illustrate the accuracy, efficiency, and applicability of the method. The results reveal that the method is very effective, straightforward, and simple.
\end{abstract}

\section{Introduction}

Integrodifferential equations (IDEs) are often involved in the mathematical formulation of physical and engineering phenomena. IDEs can be encountered in various fields of science such as physics, chemistry, biology, and engineering. These kinds of equations can also be found in numerous applications, such as electromagnetic, plasma physics, elasticity, fluid dynamics, oscillation theory, polymer rheology, chemical kinetics, biomechanics, and control theory [1-5]. Since it is usually impossible to obtain the closed-form solutions to secondorder boundary value problems of IDEs met in practice, these problems must be attacked by various approximate and numerical methods.

The purpose of this paper is to extend the application of the homotopy analysis method (HAM) to provide symbolic approximate solution for the second-order boundary 
value problems of IDEs of the following Fredholm type:

$$
u^{\prime \prime}(x)+\int_{a}^{b} K(x, s) f\left(u(s), u^{\prime}(s)\right) d s+g\left(u(x), u^{\prime}(x)\right)+h(x)=0, \quad a \leq s, x \leq b,
$$

and the following Volterra type:

$$
u^{\prime \prime}(x)+\int_{a}^{x} K(x, s) f\left(u(s), u^{\prime}(s)\right) d s+g\left(u(x), u^{\prime}(x)\right)+h(x)=0, \quad a \leq s, x \leq b,
$$

subject to the boundary conditions

$$
u(a)=\kappa_{1}, \quad u(b)=\kappa_{2},
$$

where $a, b, \kappa_{1}, \kappa_{2}$ are real finite constants, $u$ is an unknown function to be determined, $K$ and $h$ are continuous functions on $[a, b] \times[a, b]$ and $[a, b]$, respectively, and $f, g$ are nonlinear continuous functions of $u, u^{\prime}$. For details about the existence and uniqueness of solutions for such problems, see [6-8]. However, we assume that (1.1) and (1.2) subject to the boundary conditions (1.3) have a unique analytic solution on the given interval.

The numerical solvability of second-order IDEs with boundary conditions of the Fredholm and Volterra types and other related equations has been pursued by several authors. To mention a few, in [9], the authors have discussed the Legendre polynomials method for solving Fredholm equation $u^{\prime \prime}(x)+\int_{a}^{b} K(x, s) u(s) d s+f(x)+p(x) u(x)+q(x) u^{\prime}(x)=$ 0 . Furthermore, the compact finite difference method is carried out in [10] for the Volterra IDE $u^{\prime \prime}(x)+\int_{a}^{x} K(x, s) u(s) d s+g(x, u(x))=0$ and the Fredholm IDE $u^{\prime \prime}(x)+\int_{a}^{b} K(x, s) u(s) d s+$ $g(x, u(x))=0$. Recently, the monotone iterative sequences method for solving Volterra equation $u^{\prime \prime}(x)+\int_{0}^{x} K(x, s) f(u(s)) d s+g(x)=0$ is proposed in [11].

The HAM, which is proposed by Liao [12-17], is effectively and easily used to solve some classes of linear and nonlinear problems without linearization, perturbation, or discretization. The HAM is based on the homotopy, a basic concept in topology. The auxiliary parameter $\hbar$ is introduced to construct the so-called zero-order deformation equation. Thus, unlike all previous analytic techniques, the HAM provides us with a family of solution expressions in auxiliary parameter $\hbar$. As a result, the convergence region and rate of solution series are dependent upon the auxiliary parameter $\hbar$ and thus can be greatly enlarged by means of choosing a proper value of $\hbar$. This provides us with a convenient way to adjust and control convergence region and rate of solution series given by the HAM.

In the recent years, extensive work has been done using HAM, which provides analytical approximations for linear and nonlinear equations. This method has been implemented in several functional equations, such as nonlinear water waves [15], unsteady boundary-layer flows [16], solitary waves with discontinuity [17], Klein-Gordon equation [18], projectile motion with the quadratic resistance law [19], systems of fractional differential equations [20], nonlinear fractional differential equations [21, 22], systems of fractional algebraicdifferential equations [23], fractional SIR model [24], singular Volterra integral equation [25], charged particle motion for certain configurations of oscillating magnetic fields [26], Volterra population growth model [27], systems of fractional IDEs [28], and high-order IDEs [29].

The outline of the paper is as follows. In the next section, the basic idea of the HAM is introduced. In Section 3, we utilize the statement of the HAM for solving second-order 
IDEs with boundary conditions of the Fredholm and Volterra types. In Section 4, numerical examples are given to illustrate the capability of HAM. This paper ends in Section 5 with some concluding remarks.

\section{Homotopy Analysis Method}

The principles of the HAM and its applicability for various kinds of functional equations are given in [12-28]. For convenience of the reader, we will present a review of the HAM [12-17] then we will extend the HAM to construct a symbolic approximate solution to second-order IDEs with boundary conditions of the Fredholm and Volterra types. To achieve our goal, we consider the following nonlinear functional equation:

$$
N[u(x)]=0, \quad x \geq a,
$$

where $N$ is a nonlinear operator and $u(x)$ is an unknown function of independent variable $x$.

Let $u_{0}(x)$ denote an initial guess of the exact solution of (2.1), $\hbar \neq 0$ an auxiliary parameter, $H(x) \neq 0$ an auxiliary function, and $\Lambda$ an auxiliary linear operator with the property $\Lambda[f(x)]=0$ when $f(x)=0$. The auxiliary parameter $\hbar$, the auxiliary function $H(x)$, and the auxiliary linear operator $\Lambda$ play important roles within the HAM [12]. As we will see later, choosing suitable values of the auxiliary parameter $\hbar$ will help us to adjust and control the convergence region of the series solution.

Liao [12-17] constructs, using $q \in[0,1]$ as an embedding parameter, the so-called zero-order deformation equation as

$$
(1-q) \Lambda\left[\phi(x ; q)-u_{0}(x)\right]=q \hbar H(x) N[\phi(x ; q)]
$$

where $\phi(x ; q)$ is the solution of (2.1) which depends on $\hbar, H(x), \Lambda, u_{0}(x)$, and $q$. When $q=0$, the zero-order deformation equation (2.2) becomes

$$
\phi(x ; 0)=u_{0}(x)
$$

and when $q=1$, since $\hbar \neq 0$ and $H(x) \neq 0$, the zero-order deformation equation (2.2) reduces to

$$
N[\phi(x ; 1)]=0 .
$$

So, $\phi(x ; 1)$ is exactly the solution of the nonlinear equation $(2.1)$. Thus, according to (2.3) and (2.4), as $q$ increases from 0 to 1 , the solution $\phi(x ; q)$ varies continuously from the initial approximation $u_{0}(x)$ to the exact solution $u(x)$.

Define the so-called $m$ th-order deformation derivatives

$$
u_{m}(x)=\left.\frac{1}{m !} \frac{\partial^{m} \phi(x ; q)}{\partial q^{m}}\right|_{q=0},
$$


expanding $\phi(x ; q)$ in Taylor series with respect to the embedding parameter $q$, using (2.3) and (2.5), we obtain

$$
\phi(x ; q)=u_{0}(x)+\sum_{m=1}^{\infty} u_{m}(x) q^{m} .
$$

Assume that the auxiliary parameter $\hbar$, the auxiliary function $H(x)$, the initial approximation $u_{0}(x)$, and the auxiliary linear operator $\Lambda$ are properly chosen so that the power series (2.6) of $\phi(x ; q)$ converges at $q=1$. Then, we have under these assumptions the series solution $u(x)=u_{0}(x)+\sum_{m=1}^{\infty} u_{m}(x)$.

Define the vector $\vec{u}_{n}=\left\{u_{0}(x), u_{1}(x), u_{2}(x), \ldots, u_{n}(x)\right\}$. Differentiating equation (2.2) $m$-times with respect to embedding parameter $q$, then setting $q=0$ and dividing them by $m$ !. Using (2.5), we have the so-called $m$ th-order deformation equation as

$$
\Lambda\left[u_{m}(x)-\chi_{m} u_{m-1}(x)\right]=\hbar H(x) R_{m}\left(\vec{u}_{m-1}(x)\right), \quad m=1,2, \ldots, n,
$$

where

$$
\begin{gathered}
R_{m}\left(\vec{u}_{m-1}(x)\right)=\left.\frac{1}{(m-1) !} \frac{\partial^{m-1} N(\phi(x ; q))}{\partial q^{m-1}}\right|_{q=0}, \\
X_{m}= \begin{cases}0, & m \leq 1 \\
1, & m>1 .\end{cases}
\end{gathered}
$$

For any given nonlinear operator $\Lambda$, the term $R_{m}\left(\vec{u}_{m-1}(x)\right)$ can be easily expressed by (2.8). Thus, we can gain $u_{0}(x), u_{1}(x), u_{2}(x), \ldots$ by means of solving the linear high-order deformation equation (2.7) one after the other in order. The $m$ th-order approximation of $u(x)$ is given by $u(x)=\sum_{k=0}^{m-1} u_{k}(x)$.

\section{Solving Second-Order Boundary Value Problems of IDEs by HAM}

Throughout this section, we will utilize the construction for the IDEs of the Fredholm type in order not to increase the length of the paper without the loss of generality for the remaining type. However, similar construction can be implemented for the Volterra type.

Accordingly, we extend the application of the HAM to solve the second-order IDEs of the Fredholm type

$$
N[u(x)]:=u^{\prime \prime}(x)+\int_{a}^{b} K(x, s) f\left(u(s), u^{\prime}(s)\right) d s+g\left(u(x), u^{\prime}(x)\right)+h(x)=0, \quad a \leq s, x \leq b,
$$

subject to the boundary conditions (1.3).

First of all, we assume that (3.1) satisfies the initial condition $u^{\prime}(a)=\kappa$, where the unknown constant $\kappa$ can be later determined by substituting the boundary condition $u(b)=$ $\kappa_{2}$ into the obtained solution. After that, we chose the initial guess $u_{0}$ such that the initial 
conditions $u_{0}(a)=\kappa_{1}$ and $u_{0}^{\prime}(a)=\kappa$ are satisfied. That is, the initial guess will be of the form $u_{0}=u_{0}(x ; \kappa)$. Finally, we take the auxiliary linear operator $\Lambda=d^{2} / d x^{2}$ which satisfies the property $\Lambda\left[c_{1}+c_{2} t\right]=0$, where $c_{1}, c_{2}$ are constants of integrations.

The so-called zero-order deformation equation will be defined as

$$
(1-q) \frac{d^{2}}{d x^{2}}\left[\phi(x ; q, \kappa)-u_{0}(x ; \kappa)\right]=q \hbar H(x) N[\phi(x ; q, \kappa)]
$$

where

$$
\begin{aligned}
N[\phi(x ; q, \kappa)]= & \frac{d^{2} \phi(x ; q, \kappa)}{d x^{2}} \\
& +\int_{a}^{b} K(x, s) f\left(\phi(s ; q, \kappa), \phi^{\prime}(s ; q, \kappa)\right) d s+g\left(\phi(x ; q, \kappa), \phi^{\prime}(x ; q, \kappa)\right)+h(x),
\end{aligned}
$$

when $q=0$, (3.2) reduces to $\phi(x ; q, \kappa)=u_{0}(x ; \kappa)$. In this case, the so-called $m$ th-order deformation equation can be constructed as

$$
\frac{d^{2}}{d x^{2}}\left[u_{m}(x ; \kappa)-x_{m} u_{m-1}(x ; \kappa)\right]=\hbar H(x) R_{m}\left(\vec{u}_{m-1}(x ; \kappa)\right)
$$

Operating the operator $J^{2}$, the inverse operator of $d^{2} / d x^{2}$ to both sides of (3.4), then, the $m$ th-order deformation equation will have the following form:

$$
u_{m}(x ; \kappa)=x_{m} u_{m-1}(x ; \kappa)+\hbar \int_{a}^{x}\left[(x-\tau) H(\tau) R_{m}\left(\vec{u}_{m-1}(\tau ; \kappa)\right)\right] d \tau,
$$

where

$$
\begin{aligned}
R_{m}\left(\vec{u}_{m-1}(x ; \kappa)\right)= & \frac{d^{2} u_{m-1}(x ; \kappa)}{d x^{2}} \\
& +\left.\int_{a}^{b} K(x, s) \frac{1}{(m-1) !} \frac{\partial^{m-1}\left(f\left(\phi(s ; q, \kappa), \phi^{\prime}(s ; q, \kappa)\right)\right)}{\partial q^{m-1}}\right|_{q \rightarrow 0} d s \\
& +\left.\frac{1}{(m-1) !} \frac{\partial^{m-1}\left(g\left(\phi(x ; q, \kappa), \phi^{\prime}(x ; q, \kappa)\right)\right)}{\partial q^{m-1}}\right|_{q \rightarrow 0}+h(x)\left(1-x_{m}\right),
\end{aligned}
$$

and the $m$ th-order approximation of $u(x)$ can be given as

$$
u(x)=\sum_{k=0}^{m-1} u_{k}(x ; \kappa)
$$


If we substitute the boundary condition $u(b)=\kappa_{2}$ into (3.7), then we obtain an equation of the variable $\kappa$. And so, the approximate solution for (3.1) and (1.3) will be completely constructed. For simplicity, in this paper, we assume that the value of the auxiliary function is kept at $H(x)=1$.

\section{Numerical Results and Discussion}

HAM provides an analytical approximate solution in terms of an infinite power series. However, there is a practical need to evaluate this solution and to obtain numerical values from the infinite power series. The consequent series truncation and the practical procedure are conducted to accomplish this task. In this section, we consider four examples to demonstrate the performance and efficiency of the present technique. Through this paper all the symbolic and numerical computations are performed by using Mathematica 7.0 software package.

To show the accuracy of the present method for our problems, we report three types of error. The first one is the residual error, Re, defined as

$$
\begin{aligned}
\operatorname{Re}(x):=\mid \frac{d^{2}}{d x^{2}} u_{\mathrm{HAM}}^{m}(x) \\
+\int_{a}^{b} K(x, s) f\left(u_{\mathrm{HAM}}^{m}(s), \frac{d}{d s} u_{\mathrm{HAM}}^{m}(s)\right) d s \\
+g\left(u_{\mathrm{HAM}}^{m}(x), \frac{d}{d x} u_{\mathrm{HAM}}^{m}(x)\right)+h(x) \mid,
\end{aligned}
$$

for the Fredholm type and similarly for the Volterra type, while the exact, Ex, and relative, Rl, errors are defined, respectively, by

$$
\begin{aligned}
& \operatorname{Ex}(x):=\left|u_{\text {Exact }}(x)-u_{\mathrm{HAM}}^{m}(x)\right|, \\
& \operatorname{Rl}(x):=\frac{\left|u_{\text {Exact }}(x)-u_{\mathrm{HAM}}^{m}(x)\right|}{\left|u_{\text {Exact }}(x)\right|},
\end{aligned}
$$

where $s, x \in[a, b], u_{\mathrm{HAM}}^{m}$ is the $m$ th-order approximation of $u(x)$ obtained by the HAM, and $u_{\text {Exact }}$ is the exact solution.

Example 4.1. Consider the following linear Fredholm IDE:

$$
u^{\prime \prime}(x)-\int_{-1}^{1} s e^{-s} \cos x u(s) d s-2 u(x)-x=0, \quad-1 \leq s, x \leq 1
$$

subject to the boundary conditions

$$
u(-1)=-1, \quad u(1)=1
$$


The exact solution is $u(x)=c_{1} e^{\sqrt{2} x}+c_{2} e^{-\sqrt{2} x}+c_{3} \cos x-0.5 x$, where $c_{1}=0.4206 \ldots, c_{2}=$ $-0.3545 \ldots$, and $c_{3}=-0.2662 \ldots$.

According to (3.6) and (2.5), we have

$$
\begin{aligned}
R_{m}\left(\vec{u}_{m-1}(x ; \kappa)\right)= & \frac{d^{2} u_{m-1}(x ; \kappa)}{d x^{2}} \\
& -\int_{-1}^{1}\left(s e^{-s} \cos x\right) u_{m-1}(s ; \kappa) d s-2 u_{m-1}(x ; \kappa)-x\left(1-x_{m}\right)
\end{aligned}
$$

Assuming the initial approximation of (4.3) has the form $u_{0}(x)=\kappa(x+1)-1$, which satisfies the initial conditions $u_{0}(-1)=-1$ and $u_{0}^{\prime}(-1)=\kappa$. Consequently, the first few terms of the HAM series solution for (4.3) according to these initial conditions are as follows:

$$
\begin{aligned}
& u_{1}(x)=\hbar\left[0.31668-0.5311 \kappa+1.88088 x-1.12044 \kappa x+(1-\kappa) x^{2}\right. \\
& \left.-(0.16667+0.33333 \kappa) x^{3}+(0.73576+0.14313 \kappa) \cos x\right], \\
& -0.033333 \hbar^{2}[(60.81144+6.54379 \kappa)-(21.76335-17.39749 \kappa) x \\
& -(20.49956-14.06698 \kappa) x^{2}+(23.8088-1.20436 \kappa) x^{3}+5(1-\kappa) x^{4} \\
& \left.-(0.5+\kappa) x^{5}-(81.0036+0.77298 \kappa) \cos x\right] \text {. }
\end{aligned}
$$

To determine the value of $\kappa$, we must chose a value to the auxiliary parameter $\hbar$. It was proved that if we set $\hbar=-1$, then we have the Adomian decomposition solution which is a special case of the HAM solution $[30,31]$. Therefore, -1 is available value for $\hbar$. Now, substitute $\hbar=-1$ and the boundary condition at $x=1$ into the 10 th order approximation of $u(x)$ to get $\kappa=1.4818348333$.

Figure 1 shows the 10th-order approximate solution of (4.3) and (4.4) obtained by HAM at $\hbar=-1$ together with the exact solution. It is clear from the figure that the approximate solution is in high agreement with the exact solution.

Since the value of $\kappa$ has been identified previously, the valid region for the values of $\hbar$ can be obtained by the so-called $\hbar$-curve which correspond to the horizontal line as shown in Figure 2. Thus, the valid region of $\hbar$ is $-1.5<\hbar<-0.8$.

Figure 3 shows a comparison between the exact and residual errors obtained from the 10th-order approximation of $u(x)$ for (4.3) and (4.4) at $\hbar=-1$, while Figures 4 and 5 show respectively how the exact and residual errors change with varying the values of $\hbar$. 


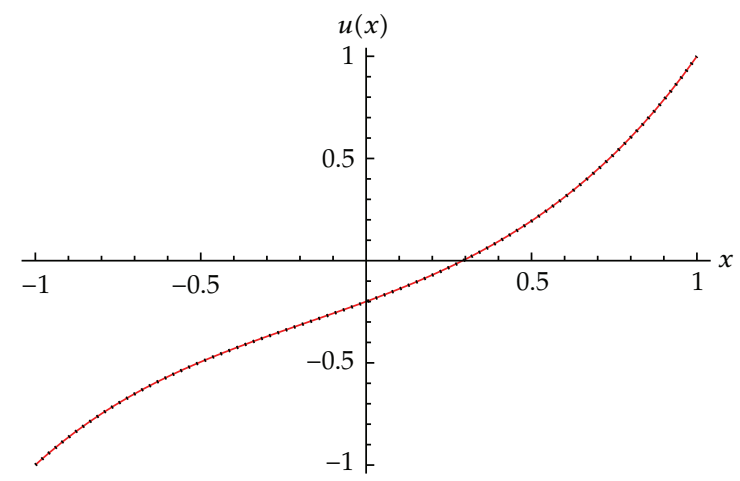

Figure 1: Solutions of (4.3) and (4.4). Dotted line: the 10th-order approximate HAM solution at $\hbar=-1$ and solid line: the exact solution.

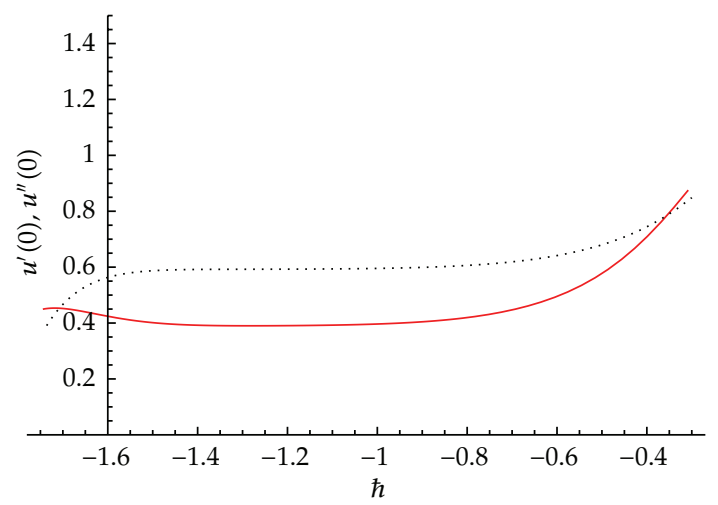

Figure 2: The $\hbar$-curves of $u^{\prime}(0)$ and $u^{\prime \prime}(0)$ which are corresponding to the 10th-order approximate HAM solution of (4.3) and (4.4). Dotted line: $u^{\prime}(0)$ and solid line: $u^{\prime \prime}(0)$.

Example 4.2. Consider the following nonlinear Fredholm IDE:

$$
u^{\prime \prime}(x)-\int_{0}^{1}(x-s)^{2} e^{u(s)} d s+\left(u^{\prime}(x)\right)^{2}+\left(\frac{3}{2} x^{2}-\frac{5}{3} x+\frac{7}{12}\right)=0, \quad 0 \leq s, x \leq 1
$$

subject to the boundary conditions

$$
u(0)=0, \quad u(1)=\ln 2 .
$$

The exact solution is $u(x)=\ln (x+1)$. 


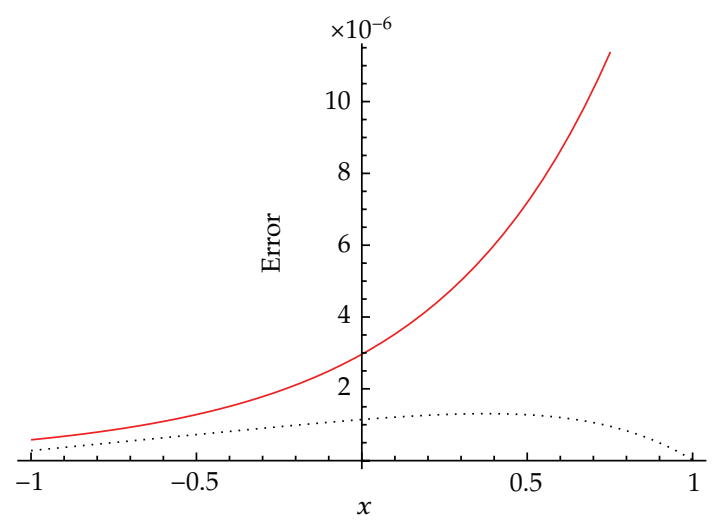

Figure 3: Errors of (4.3) and (4.4) at $\hbar=-1$. Dotted line: the exact error and solid line: the residual error.

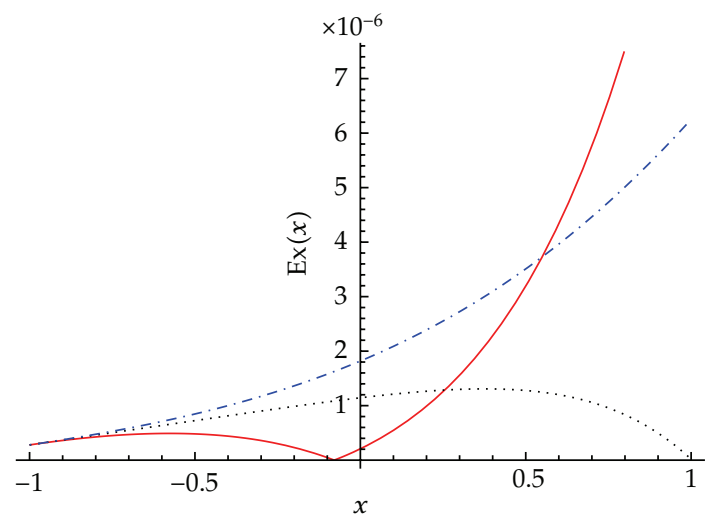

Figure 4: Exact error of (4.3) and (4.4) at different values of $\hbar$. Dash-dotted line: $\hbar=-1.1$, dotted line: $\hbar=-1$, and solid line: $\hbar=-0.9$.

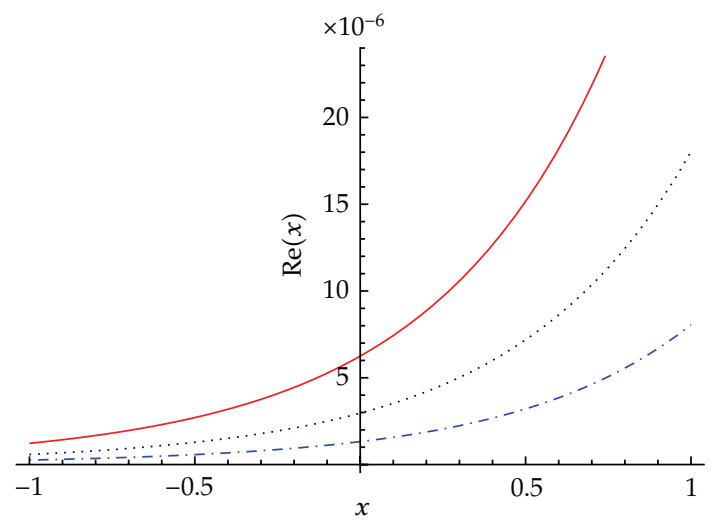

Figure 5: Residual error of (4.3) and (4.4) at different values of $\hbar$. Dash-dotted line: $\hbar=-1.1$, dotted line: $\hbar=-1$, and solid line: $\hbar=-0.9$. 
According to (3.6) and (2.5), we have

$$
\begin{aligned}
R_{m}\left(\vec{u}_{m-1}(x ; \kappa)\right)= & \frac{d^{2}}{d x^{2}} u_{m-1}(x ; \kappa)+\left(\frac{3}{2} x^{2}-\frac{5}{3} x+\frac{7}{12}\right)\left(1-x_{m}\right) \\
& -\left.\int_{0}^{1}(x-s)^{2} \frac{1}{(m-1) !} \frac{\partial^{m-1}(\exp (\phi(s ; q, \kappa)))}{\partial q^{m-1}}\right|_{q \rightarrow 0} d s \\
& +\sum_{i=0}^{m-1} \frac{d}{d x} u_{i}(x ; \kappa) \frac{d}{d x} u_{m-1-i}(x ; \kappa) .
\end{aligned}
$$

Choose the initial guess approximation as $u_{0}(x)=k x$, which satisfies the initial conditions $u_{0}(0)=0$ and $u_{0}^{\prime}(0)=\kappa$. Then, we have the following approximations terms of the HAM series solution for (4.8):

$$
\begin{aligned}
& u_{1}(x)=\frac{h x^{2}}{72 \kappa^{3}}\left(72-72 e^{\kappa}+72 e^{\kappa} \kappa+24 x \kappa-24 e^{\kappa} x \kappa-36 e^{\kappa} \kappa^{2}\right. \\
& \left.+24 e^{\kappa} x \kappa^{2}+6 x^{2} \kappa^{2}-6 e^{\kappa} x^{2} \kappa^{2}+21 \kappa^{3}-20 x \kappa^{3}+9 x^{2} \kappa^{3}+36 \kappa^{5}\right), \\
& u_{2}(x)=u_{1}(x)+\frac{h^{2} x^{2}}{2160 \kappa^{8}}\left(47520-95040 e^{\kappa}+47520 e^{2 \kappa}+97200 \kappa-66960 e^{\kappa} \mathcal{\kappa}-30240 e^{2 \kappa} \mathcal{\kappa}\right. \\
& +5760 x \mathcal{K}-11520 e^{\kappa} x \mathcal{\kappa}+5760 e^{2 \kappa} x \mathcal{\kappa}+36000 \kappa^{2}+24480 e^{\kappa} \kappa^{2} \\
& +19440 e^{2 \kappa} \kappa^{2}+10800 x \kappa^{2}-6480 e^{\kappa} x \kappa^{2}-4320 e^{2 \kappa} x \kappa^{2}+360 x^{2} \kappa^{2} \\
& +360 e^{2 \kappa} x^{2} \kappa^{2}-720 e^{\kappa} x^{2} \kappa^{2}+7560 \kappa^{3}-12240 e^{\kappa} \mathcal{\kappa}^{3}-12240 e^{2 \kappa} \mathcal{\kappa}^{3} \\
& +4800 x \kappa^{3}+960 e^{\kappa} x \kappa^{3}+3600 e^{2 \kappa} x \kappa^{3}+540 x^{2} \kappa^{3}-180 e^{\kappa} x^{2} \kappa^{3} \\
& -360 e^{2 \kappa} x^{2} \kappa^{3}+3780 e^{\kappa} \kappa^{4}+5580 e^{2 \kappa} \mathcal{K}^{4}+1260 x \kappa^{4}-900 e^{\kappa} x \kappa^{4} \\
& -2400 e^{2 \kappa} x \kappa^{4}+300 x^{2} \kappa^{4}-120 e^{\kappa} x^{2} \mathcal{K}^{4}+360 e^{2 \kappa} x^{2} \mathcal{K}^{4}+15120 \kappa^{5} \\
& -16770 e^{\kappa} \mathcal{K}^{5}-1620 e^{2 \kappa} \mathcal{K}^{5}+600 e^{\kappa} x \kappa^{5}+900 e^{2 \kappa} x \kappa^{5}+105 x^{2} \kappa^{5} \\
& -75 e^{\kappa} x^{2} \mathcal{\kappa}^{5}-180 e^{2 \kappa} x^{2} \mathcal{\kappa}^{5}+15600 e^{\kappa} \kappa^{6}+270 e^{2 \kappa} \mathcal{K}^{6}+4320 x \kappa^{6} \\
& -4540 e^{\kappa} x \kappa^{6}-180 e^{2 \kappa} x \kappa^{6}+30 e^{\kappa} x^{2} \kappa^{6}+45 e^{2 \kappa} x^{2} \kappa^{6}-7710 e^{\kappa} \kappa^{7} \\
& +4420 e^{\kappa} x \kappa^{7}+720 x^{2} \kappa^{7}-745 e^{\kappa} x^{2} \kappa^{7}+630 \kappa^{8}+2160 e^{\kappa} \kappa^{8} \\
& -600 x \kappa^{8}-1800 e^{\kappa} x \kappa^{8}+270 x^{2} \kappa^{8}+540 e^{\kappa} x^{2} \kappa^{8}+72 x^{3} \kappa^{8} \\
& -72 e^{\kappa} x^{3} \kappa^{8}-540 e^{\kappa} \mathcal{K}^{9}+420 x \kappa^{9}+360 e^{\kappa} x \kappa^{9}-300 x^{2} \kappa^{9} \\
& \left.-90 e^{\kappa} x^{2} \kappa^{9}+108 x^{3} \kappa^{9}+1080 \kappa^{10}+720 x \kappa^{11}\right) .
\end{aligned}
$$




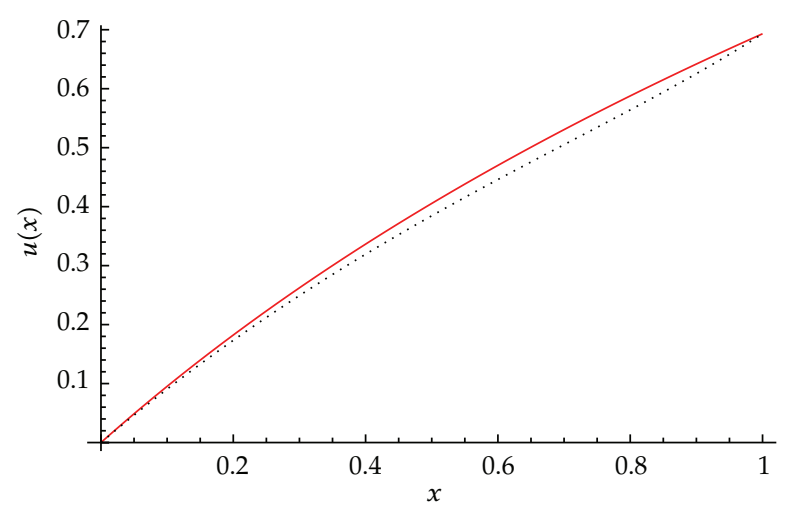

Figure 6: Solutions of (4.8) and (4.9). Dotted line: the 5th-order approximate HAM solution at $\hbar=-1$ and solid line: the exact solution.

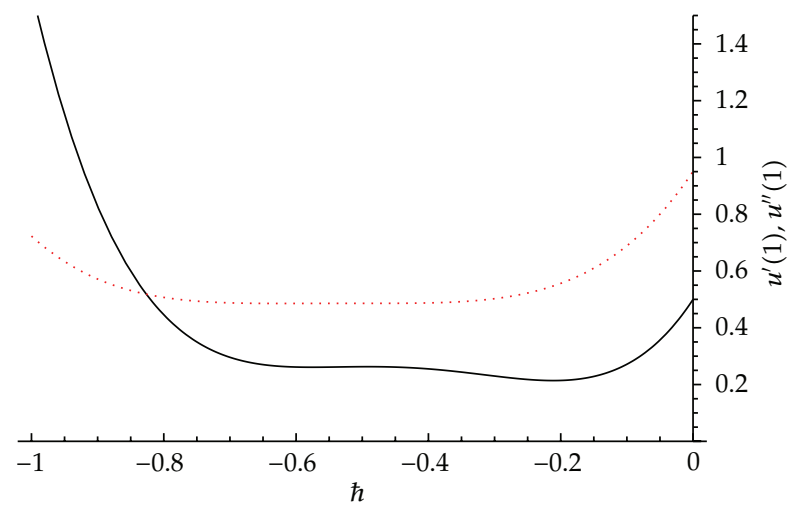

Figure 7: The $\hbar$-curves of $u^{\prime}(1)$ and $u^{\prime \prime}(1)$ which are corresponding to the 5th-order approximate HAM solution of (4.8) and (4.9). Dotted line: $u^{\prime}(1)$ and solid line: $u^{\prime \prime}(1)$.

As in the previous example, we determine an introductory value of the constant $\kappa$ by substituting $\hbar=-1$ and the boundary condition at $x=1$ into the obtained HAM solution. So, the 5th-order approximation of $u(x)$ gives $\kappa=0.9507338904$. Figure 6 shows the 5 th-order approximate solution of (4.8) and (4.9) obtained by HAM at $\hbar=-1$ together with the exact solution, while Figure 7 shows the $\hbar$-curve corresponding to the 5 th-order approximate HAM solution. It is clear from Figure 7 that the valid region of $\hbar$ is $-0.7<\hbar<-0.4$.

Our next goal is to show how the auxiliary parameter $\hbar$ affects the approximate solutions. However, it is evident from Figure 6 that both curves corresponding to the 5thorder approximate HAM solution and the exact solution are not coinciding in general at $\hbar=-1$. To improve the solution, let us compute other values of $\kappa$ at different values of $\hbar$. Table 1 shows the values of the constant $\kappa$ at different values of the auxiliary parameter $\hbar$.

Representation the exact solution and the 5th-order approximate HAM solution of (4.8) and (4.9) at different values of $\hbar$ mentioned in Table 1 shows that all these solutions are approximately identical. However, Figure 8 shows the correspondence between the exact solution and the 5th-order approximate HAM solution of (4.8) and (4.9) at $\hbar=-0.6$. From the last mentioned figure, we see that we can achieve a good approximation with the exact solution by using a few terms in HAM. 
Table 1: The values of $\kappa$ at different values of $\hbar$.

\begin{tabular}{lc}
\hline$\hbar$ & $\kappa$ \\
\hline-0.8 & 0.9973778496 \\
-0.6 & 0.9987971735 \\
-0.4 & 0.9834454136 \\
\hline
\end{tabular}

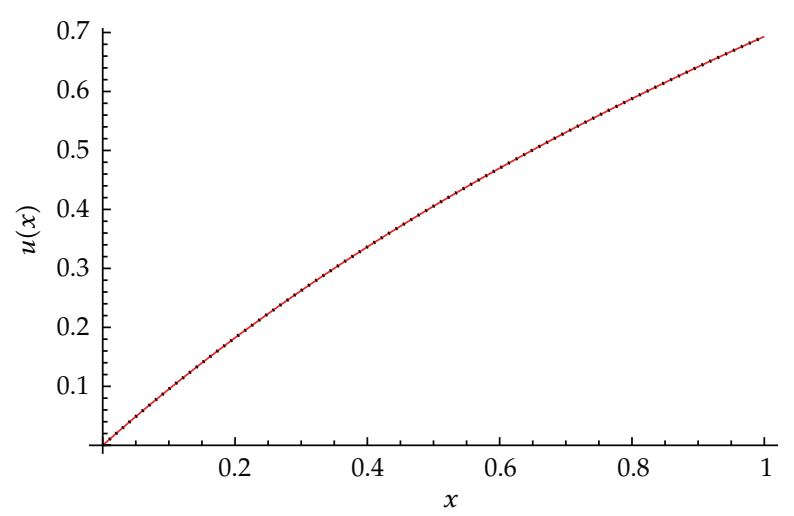

Figure 8: Solutions of (4.8) and (4.9). Dotted line: the 5th-order approximate HAM solution at $\hbar=-0.6$ and solid line: the exact solution.

Figure 9 shows a comparison between the exact errors of (4.8) and (4.9) at different values of $\hbar$ corresponding to the 5 th-order approximate HAM solution. It is to be noted that the accuracy of certain node at $\hbar=-0.6$ is more convincing than the other values of $\hbar$.

Example 4.3. Consider the following linear Volterra IDE:

$$
u^{\prime \prime}(x)-\int_{0}^{x} e^{-s} \sin x u^{\prime}(s) d s+u(x)-\left(\frac{1}{2} e^{-x} \sin 2 x-\sin x\right)=0, \quad 0 \leq s, x \leq \frac{\pi}{2}
$$

subject to the boundary conditions

$$
u(0)=-1, \quad u\left(\frac{\pi}{2}\right)=1
$$

The exact solution is $u(x)=\sin x-\cos x$.

According to (3.6) and (2.5), we have

$$
\begin{aligned}
R_{m}\left(\vec{u}_{m-1}(x ; \kappa)\right)= & \frac{d^{2}}{d x^{2}} u_{m-1}(x ; \kappa)-\int_{0}^{x} \sin x e^{-s}\left(\frac{d}{d s} u_{m-1}(s ; \kappa)\right) d s+u_{m-1}(x ; \kappa) \\
& -\left(\frac{1}{2} e^{-x} \sin 2 x-\sin x\right)\left(1-x_{m}\right) .
\end{aligned}
$$




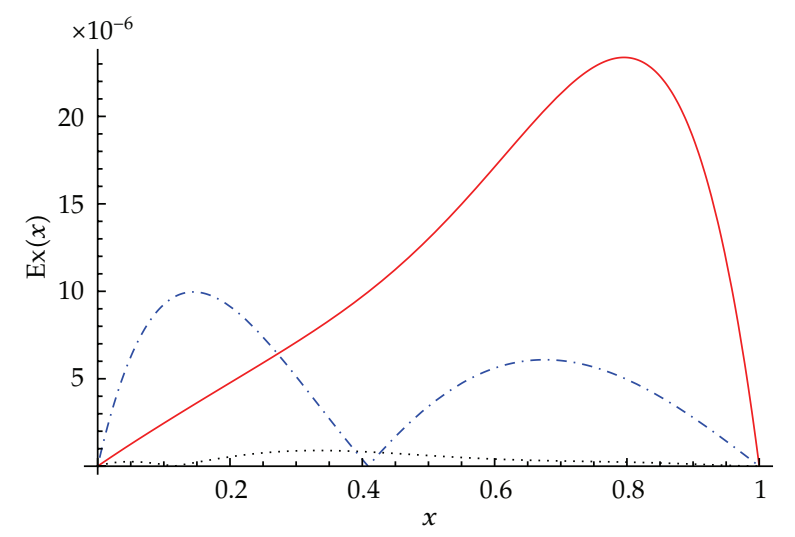

Figure 9: Exact error of (4.8) and (4.9) at different values of $\hbar$. Dash-dotted line: $\hbar=-0.4$, dotted line: $\hbar=-0.6$, and solid line: $\hbar=-0.8$.

Choose the initial guess approximation as $u_{0}(x)=\kappa x-1$, which satisfies the initial conditions $u_{0}(0)=-1$ and $u_{0}^{\prime}(0)=\kappa$. Using the iteration formula (3.5), we can directly obtain the following approximations terms of the HAM series solution for (4.13):

$$
\begin{aligned}
u_{1}(x)=\frac{1}{150} \hbar( & 12-75 \kappa+120 x-75 \kappa x-75 x^{2}+25 \kappa x^{3}+75 \kappa e^{-x} \cos x \\
& \left.-12 e^{-x} \cos 2 x-150 \sin x+150 \kappa \sin x+9 e^{-x} \sin 2 x\right), \\
u_{2}(x)=u_{1}(x)+\frac{1}{1200} \hbar^{2} & \left(\frac{2159856}{4225}+300 e^{-x}-\frac{5376 x}{65}-552 x^{2}+160 x^{3}-50 x^{4}-\frac{2349 \kappa}{2}\right. \\
& -15 e^{-2 x} \kappa-300 e^{-x} \kappa+1080 x \kappa-300 x^{2} \kappa+100 x^{3} \kappa+10 x^{5} \kappa \\
+ & \frac{3}{50} e^{-2 x}\left(-375 \kappa+8 e^{x}(25 \kappa-177)\right) \cos 2 x+\frac{465}{169} e^{-2 x} \cos 3 x-750 \sin x \\
& -3 e^{-2 x} \sin x+600 e^{-x} \sin x+840 \kappa \sin x-1800 e^{-x} \kappa \sin x-600 e^{-x} x \kappa \sin x \\
& -3 e^{-2 x} \cos x\left(3-20 e^{x}\left(-12-10 x+25 \kappa+20 x \kappa+5 x^{2} \kappa\right)+5 \kappa \sin x\right) \\
+ & \left.\frac{4068}{25} e^{-x} \sin 2 x-84 e^{-x} \kappa \sin 2 x-\frac{405}{169} e^{-2 x} \sin 3 x\right) .
\end{aligned}
$$

The rest of components of the iteration formula (3.5) can be obtained in a similar way.

To find the value of $\mathcal{\kappa}$, substitute $\hbar=-1$ and the boundary condition at $x=\pi / 2$ into the 5 th-order approximation of $u(x)$ to get $\kappa=1.0000000668$. After that, substituting the value of $\mathcal{\kappa}$ in the 5 th-order approximation of $u(x)$ and plotting the $\hbar$-curve, we can obtain the valid region of $\hbar$ which is $-1.3<\hbar<-0.7$ as shown in Figure 10 .

The graph of the 5th-order approximate HAM solution of (4.13) and (4.14) at $\hbar=-1$ together with the exact solution are depicted in Figure 11. It is evident from the figure that the 5 th-order approximate HAM solution agrees with the exact solution in the interval $[0, \pi / 2]$. 


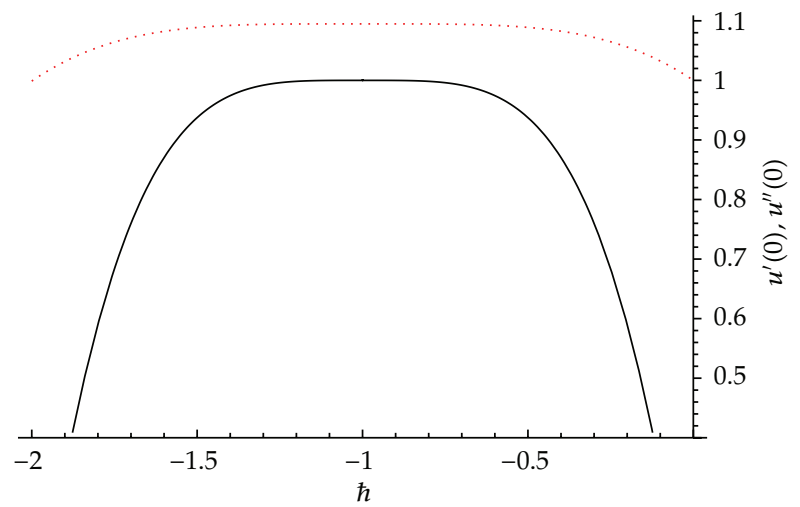

Figure 10: The $\hbar$-curves of $u^{\prime}(0)$ and $u^{\prime \prime}(0)$ which are corresponding to the 5th-order approximate HAM solution of (4.13) and (4.14). Dotted line: $u^{\prime}(0)$ and solid line: $u^{\prime \prime}(0)$.

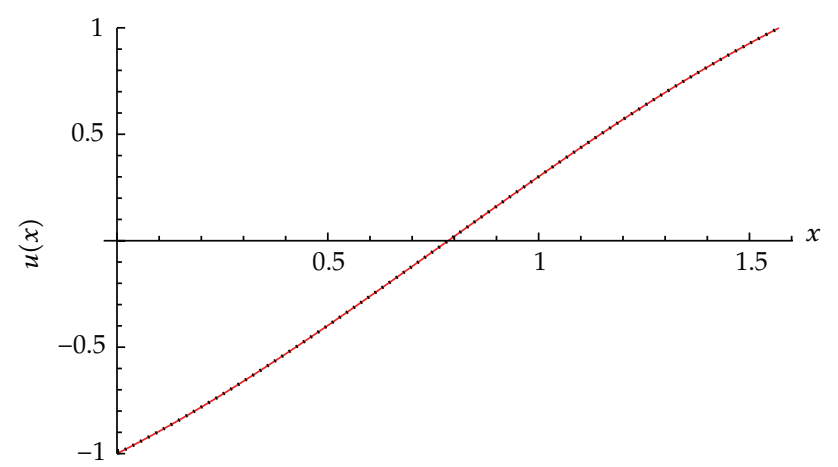

Figure 11: Solutions of (4.13) and (4.14). Dotted line: the 5th-order approximate HAM solution at $\hbar=-1$ and solid line: the exact solution.

In Table 2, the exact, relative, and residual errors have been calculated for various $x$ in $[0, \pi / 2]$ to measure the extent of agreement between the 5th-order approximate HAM solution at $\hbar=-1$ and the exact solution. From the table, it can be seen that the HAM provides us with the accurate approximate solution of (4.13) and (4.14).

Example 4.4. Consider the following nonlinear Volterra IDE:

$$
u^{\prime \prime}(x)+\int_{0}^{x}(u(s))^{2} d s+\left(\frac{x}{2}-\sinh x-\frac{1}{4} \sinh 2 x\right)=0, \quad 0 \leq s, x \leq 1
$$

subject to the boundary conditions

$$
u(0)=0, \quad u(1)=\sinh (1)
$$

The exact solution is $u(x)=\sinh x$. 
Table 2: Exact, relative, and residual errors of (4.13) and (4.14) at $\hbar=-1$ and $\kappa=1.0000000668$.

\begin{tabular}{lccc}
\hline$x$ & Exact error & Relative error & Residual Error \\
\hline 0.1 & $4.973784 \times 10^{-10}$ & $5.556241 \times 10^{-10}$ & $1.112207 \times 10^{-9}$ \\
0.2 & $2.426173 \times 10^{-11}$ & $3.104917 \times 10^{-11}$ & $5.782878 \times 10^{-9}$ \\
0.3 & $3.202272 \times 10^{-12}$ & $4.853278 \times 10^{-12}$ & $5.440901 \times 10^{-7}$ \\
0.4 & $1.244961 \times 10^{-11}$ & $2.341725 \times 10^{-11}$ & $2.572090 \times 10^{-6}$ \\
0.5 & $1.030532 \times 10^{-10}$ & $2.588255 \times 10^{-10}$ & $8.420042 \times 10^{-6}$ \\
0.6 & $5.499366 \times 10^{-10}$ & $2.109517 \times 10^{-9}$ & $2.202234 \times 10^{-5}$ \\
0.7 & $2.259143 \times 10^{-9}$ & $1.872873 \times 10^{-8}$ & $4.964877 \times 10^{-5}$ \\
0.8 & $7.674839 \times 10^{-9}$ & $3.716740 \times 10^{-7}$ & $1.008886 \times 10^{-4}$ \\
0.9 & $2.263322 \times 10^{-8}$ & $1.399558 \times 10^{-7}$ & $1.900279 \times 10^{-4}$ \\
1.0 & $5.985469 \times 10^{-8}$ & $1.987414 \times 10^{-7}$ & $3.378840 \times 10^{-4}$ \\
$\vdots$ & $\vdots$ & $\vdots$ & $\vdots$ \\
$\pi / 2$ & $4.370067 \times 10^{-6}$ & $0.370067 \times 10^{-6}$ & $4.598268 \times 10^{-3}$ \\
\hline
\end{tabular}

According to (3.6) and (2.5), we have

$$
\begin{aligned}
R_{m}\left(\vec{u}_{m-1}(x ; \kappa)\right)= & \frac{d^{2}}{d x^{2}} u_{m-1}(x ; \kappa)+\left(\frac{x}{2}-\sinh x-\frac{1}{4} \sinh 2 x\right)\left(1-x_{m}\right) \\
& +\int_{0}^{x}\left(\sum_{j=0}^{m-1} u_{j}(s ; \kappa) u_{m-1-j}(s ; \kappa)\right) d s .
\end{aligned}
$$

Assume that the initial approximation of (4.18) has the form $u_{0}(x)=\kappa x$, which satisfies the initial conditions $u_{0}(0)=0$ and $u_{0}^{\prime}(0)=\kappa$. Using the iteration formula (3.5), we can directly obtain the following approximations terms of the HAM series solution for (4.18) subject to these initial conditions:

$$
\begin{gathered}
u_{1}(x)=\frac{1}{120} \hbar\left(x\left(135+10 x^{2}+2 x^{4} \kappa^{2}\right)-15(8+\cosh x) \sinh x\right), \\
u_{2}(x)=u_{1}(x)+\frac{1}{3360} \hbar^{2}\left(3780 x+280 x^{3}-13545 x \kappa+126 x^{5} \kappa+\frac{8}{3} x^{7} \kappa\right. \\
+56 x^{5} \kappa^{2}+\frac{2}{9} x^{9} \kappa^{3}-6720 x \kappa \cosh x-\frac{105}{2} x \kappa \cosh 2 x-3360 \sinh x \\
\left.+20160 \kappa \sinh x-210 \sinh 2 x+\frac{315}{4} \kappa \sinh 2 x\right) .
\end{gathered}
$$

Similarly to the previous discussion, one can substitute $\hbar=-1$ and the boundary condition at $x=1$ into the 5 th-order approximation of $u(x)$ to get $\kappa=1.0000000063$. Figure 12 shows the agreement between the 5th-order approximate HAM solution of (4.18) and (4.19) at $\hbar=-1$ and the exact solution. 


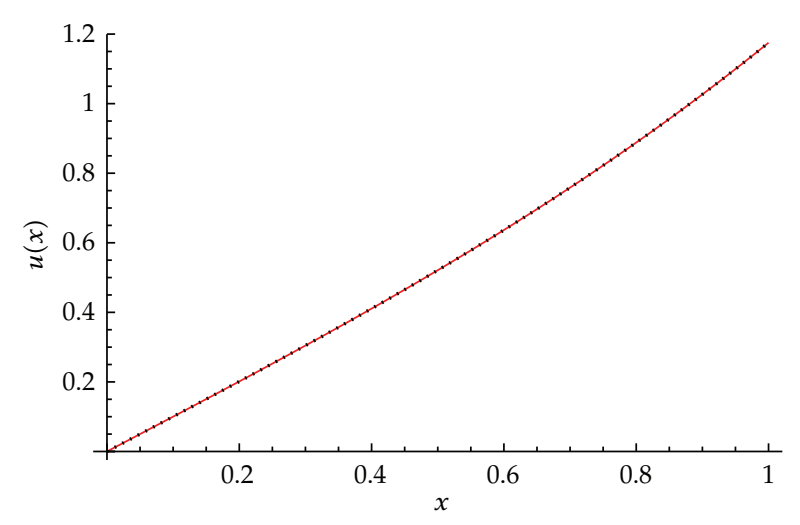

Figure 12: Solutions of (4.18) and (4.19). Dotted line: the 5th-order approximate HAM solution at $\hbar=-1$ and solid line: the exact solution.

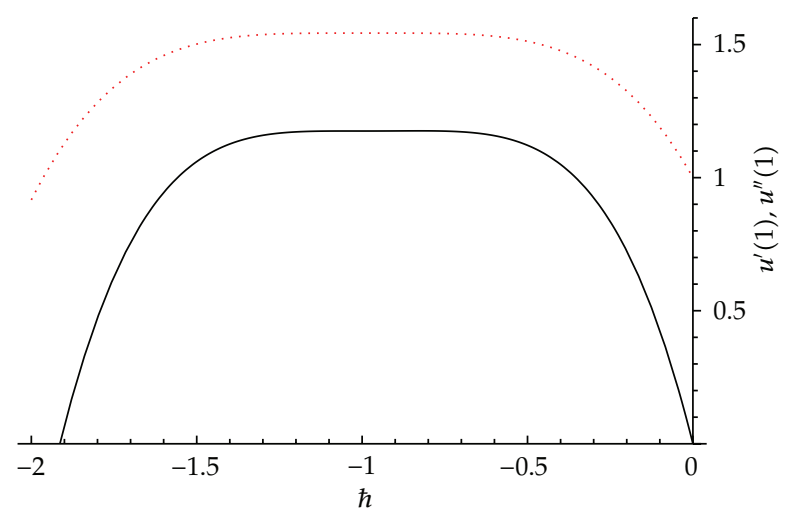

Figure 13: The $\hbar$-curves of $u^{\prime}(1)$ and $u^{\prime \prime}(1)$ which are corresponding to the 5th-order approximate HAM solution of (4.18) and (4.19). Dotted line: $u^{\prime}(1)$ and solid line: $u^{\prime \prime}(1)$.

Since the value of $\kappa$ has been identified previously, the valid region for the values of $\hbar$ can be obtained by the so-called $\hbar$-curve which correspond to the horizontal line as shown in Figure 13. Thus, it is clear from the figure that the valid region of $\hbar$ is $-1.2<\hbar<-0.7$.

The detailed data of $u(x)$ for (4.18) and (4.19) that includes the exact nodal values, the 5 th-order approximate HAM solution nodal values, and the exact error are given in Table 3. It is clear that the accuracy obtained using HAM is in advanced by using only five terms approximations.

\section{Conclusion}

The main concern of this work has been to propose an efficient algorithm for the solution of second-order IDEs with boundary conditions of the Fredholm and Volterra types. The goal has been achieved by extending the HAM to solve this class of boundary value problems. We can conclude that the HAM is a powerful and efficient technique in finding approximate solutions for linear and nonlinear boundary value problems of second-order IDEs of different types. The proposed algorithm produced a rapidly convergent series by choosing suitable 
Table 3: Numerical results of (4.18) and (4.19) at $\hbar=-1$ and $\kappa=1.0000000063$.

\begin{tabular}{lccc}
\hline$x$ & Exact solution & HAM solution & Exact error \\
\hline 0.1 & 0.1001667500 & 0.1001667500 & $5.760464 \times 10^{-14}$ \\
0.2 & 0.2013360025 & 0.2013360025 & $2.119789 \times 10^{-13}$ \\
0.3 & 0.3045202934 & 0.3045202934 & $5.767938 \times 10^{-13}$ \\
0.4 & 0.4107523258 & 0.4107523258 & $8.997664 \times 10^{-14}$ \\
0.5 & 0.5210953055 & 0.5210953055 & $2.401551 \times 10^{-13}$ \\
0.6 & 0.6366535821 & 0.6366535821 & $1.919631 \times 10^{-12}$ \\
0.7 & 0.7585837018 & 0.7585837018 & $3.099931 \times 10^{-11}$ \\
0.8 & 0.8881059822 & 0.8881059820 & $2.174120 \times 10^{-10}$ \\
0.9 & 1.0265167257 & 1.0265167244 & $1.282535 \times 10^{-9}$ \\
1.0 & 1.1752011936 & 1.1752011876 & $6.091291 \times 10^{-9}$ \\
\hline
\end{tabular}

values of the auxiliary parameter $\hbar$. After computing several approximants and using the boundary conditions at the boundary points, we can easily determine the approximate solution.

There are two important points to make here. First, HAM provides us with a simple way to adjust and control the convergence region of the series solution by introducing the auxiliary parameter $\hbar$. Second, the results obtained by HAM are very effective and convenient in linear and nonlinear cases with less computational work. This confirms our belief that the efficiency of our technique gives it much wider applicability for general classes of linear and nonlinear problems.

\section{References}

[1] R. P. Kanwal, Linear Integral Differential Equations: Theory and Technique, Birkhauser, Boston, Ga, USA, 2nd edition, 1996.

[2] F. Bloom, "Asymptotic bounds for solutions to a system of damped integro-differential equations of electromagnetic theory," Journal of Mathematical Analysis and Applications, vol. 73, no. 2, pp. 524-542, 1980.

[3] K. Holmåker, "Global asymptotic stability for a stationary solution of a system of integro-differential equations describing the formation of liver zones," SIAM Journal on Mathematical Analysis, vol. 24, no. 1, pp. 116-128, 1993.

[4] L. K. Forbes, S. Crozier, and D. M. Doddrell, "Calculating current densities and fields produced by shielded magnetic resonance imaging probes," SIAM Journal on Applied Mathematics, vol. 57, no. 2, pp. 401-425, 1997.

[5] A. J. Jerri, Introduction to Integral Equations with Applications, John Wiley \& Sons, New York, NY, USA, 2nd edition, 1999.

[6] R. P. Agarwal, "Boundary value problems for higher order integro-differential equations," Nonlinear Analysis, vol. 7, no. 3, pp. 259-270, 1983.

[7] R. P. Agarwal, Boundary Value Problems for High Ordinary Differential Equations, World Scientific, Singapore, 1986.

[8] Z. Wang, L. Liu, and Y. Wu, "The unique solution of boundary value problems for nonlinear secondorder integral-differential equations of mixed type in Banach spaces," Computers \& Mathematics with Applications, vol. 54, no. 9-10, pp. 1293-1301, 2007.

[9] A. Saadatmandi and M. Dehghan, "Numerical solution of the higher-order linear Fredholm integrodifferential-difference equation with variable coefficients," Computers $\mathcal{E}$ Mathematics with Applications, vol. 59, no. 8, pp. 2996-3004, 2010.

[10] J. Zhao and R. M. Corless, "Compact finite difference method for integro-differential equations," Applied Mathematics \& Computation, vol. 177, no. 1, pp. 271-288, 2006. 
[11] Q. M. Al-Mdallal, "Monotone iterative sequences for nonlinear integro-differential equations of second order," Nonlinear Analysis, vol. 12, no. 6, pp. 3665-3673, 2011.

[12] S. J. Liao, Beyond Perturbation: Introduction to the Homotopy Analysis Methods, Chapman \& Hall, Boca Raton, Fla, USA, 2003.

[13] S. Liao, "On the homotopy analysis method for nonlinear problems," Applied Mathematics E Computation, vol. 147, no. 2, pp. 499-513, 2004.

[14] S. J. Liao, "Homotopy analysis method: a new analytic method for nonlinear problems," Applied Mathematics E Mechanics, vol. 19, no. 10, pp. 957-962, 1998.

[15] S. J. Liao and K. F. Cheung, "Homotopy analysis of nonlinear progressive waves in deep water," Journal of Engineering Mathematics, vol. 45, no. 2, pp. 105-116, 2003.

[16] S. Liao, "Series solutions of unsteady boundary-layer flows over a stretching flat plate," Studies in Applied Mathematics, vol. 117, no. 3, pp. 239-263, 2006.

[17] W. Wu and S. J. Liao, "Solving solitary waves with discontinuity by means of the homotopy analysis method," Chaos, Solitons and Fractals, vol. 26, no. 1, pp. 177-185, 2005.

[18] Q. Sun, "Solving the Klein-Gordon equation by means of the homotopy analysis method," Applied Mathematics \& Computation, vol. 169, no. 1, pp. 355-365, 2005.

[19] K. Yabushita, M. Yamashita, and K. Tsuboi, "An analytic solution of projectile motion with the quadratic resistance law using the homotopy analysis method," Journal of Physics A, vol. 40, no. 29, pp. 8403-8416, 2007.

[20] M. Zurigat, S. Momani, Z. Odibat, and A. Alawneh, "The homotopy analysis method for handling systems of fractional differential equations," Applied Mathematical Modelling, vol. 34, no. 1, pp. 24-35, 2010.

[21] Z. Odibat, S. Momani, and H. Xu, "A reliable algorithm of homotopy analysis method for solving nonlinear fractional differential equations," Applied Mathematical Modelling, vol. 34, no. 3, pp. 593-600, 2010.

[22] I. Hashim, O. Abdulaziz, and S. Momani, "Homotopy analysis method for fractional IVPs," Communications in Nonlinear Science and Numerical Simulation, vol. 14, no. 3, pp. 674-684, 2009.

[23] M. Zurigat, S. Momani, and A. Alawneh, "Analytical approximate solutions of systems of fractional algebraic-differential equations by homotopy analysis method," Computers $\mathcal{E}$ Mathematics with Applications, vol. 59, no. 3, pp. 1227-1235, 2010.

[24] O. Abu Arqub and A. El-Ajou, "Solution of the fractional epidemic model by homotopy analysis method," Journal of King Saud University. In press.

[25] H. Vosughi, E. Shivanian, and S. Abbasbandy, "A new analytical technique to solve Volterra's integral equations," Mathematical Methods in the Applied Sciences, vol. 34, no. 10, pp. 1243-1253, 2011.

[26] H.S. Nik, S. Effati, and R. Buzhabadi, "Analytic-approximate solution for an integro-differential equation arising in oscillating magnetic fields using homotopy analysis method," Iranian Journal of Optimization, vol. 2, no. 3, pp. 518-535, 2010.

[27] N. A. Khan, A. Ara, and M. Jamil, "Approximations of the nonlinear Volterra's population model by an efficient numerical method," Mathematical Methods in the Applied Sciences, vol. 34, no. 14, pp. 1733-1738, 2011.

[28] M. Zurigat, S. Momani, and A. Alawneh, "Homotopy analysis method for systems of fractional integro-differential equations," Neural, Parallel E Scientific Computations, vol. 17, no. 2, pp. 169-186, 2009.

[29] H. M. Jaradat, F. Awawdeh, and O. Alsayyed, "Series solution to the high-order integro-differential equations," Analele Universităţii din Oradea-Fascicola Matematică, vol. 16, pp. 247-257, 2009, Tom XVI.

[30] J. Cang, Y. Tan, H. Xu, and S. J. Liao, "Series solutions of non-linear Riccati differential equations with fractional order," Chaos, Solitons and Fractals, vol. 40, no. 1, pp. 1-9, 2009.

[31] F. M. Allan, "Derivation of the Adomian decomposition method using the homotopy analysis method," Applied Mathematics E Computation, vol. 190, no. 1, pp. 6-14, 2007. 


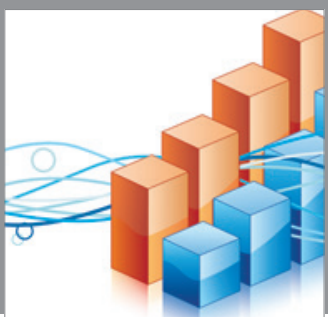

Advances in

Operations Research

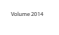

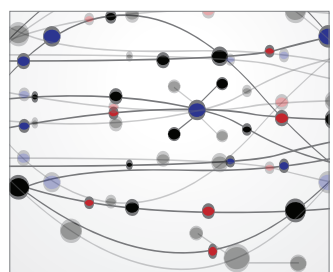

\section{The Scientific} World Journal
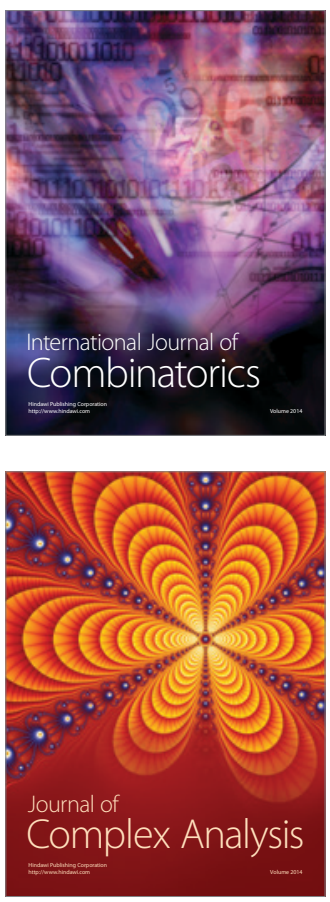

International Journal of

Mathematics and

Mathematical

Sciences
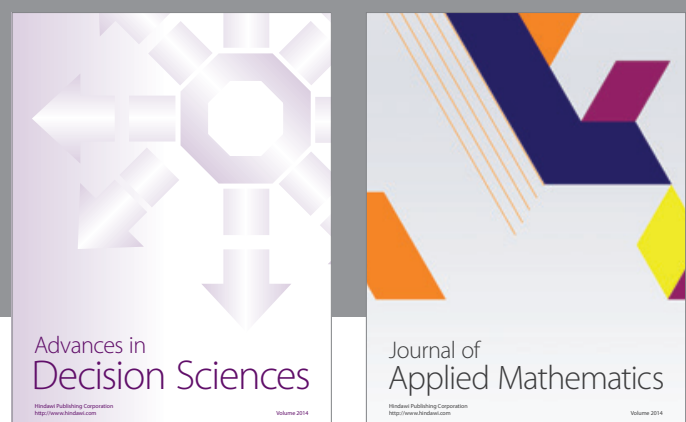

Journal of

Applied Mathematics
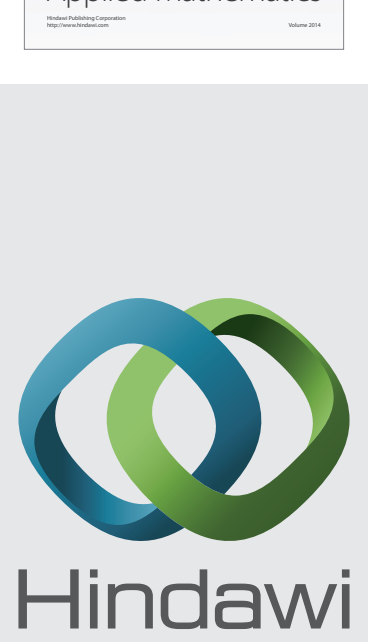

Submit your manuscripts at http://www.hindawi.com
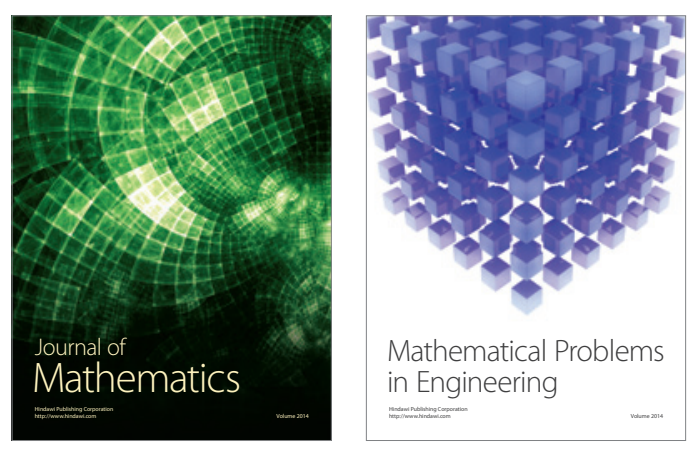

Mathematical Problems in Engineering
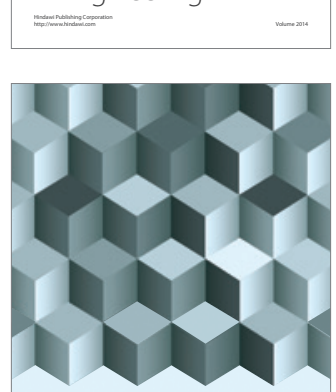

Journal of

Function Spaces
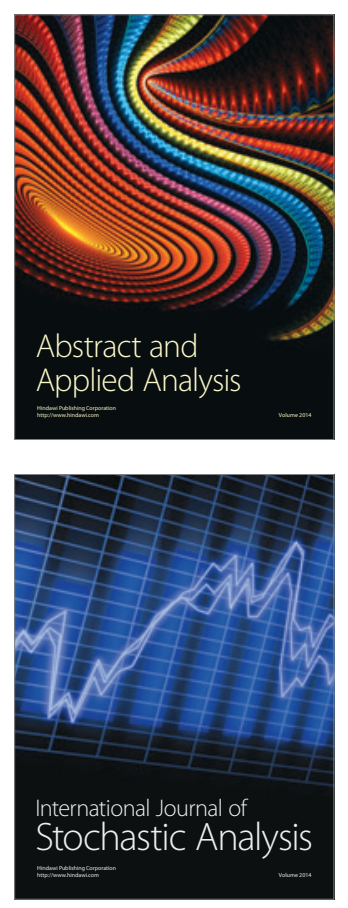

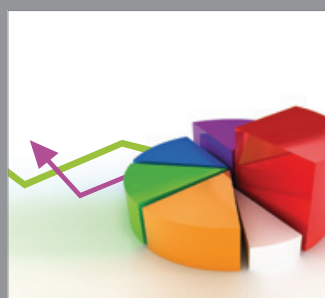

ournal of

Probability and Statistics

Promensencen
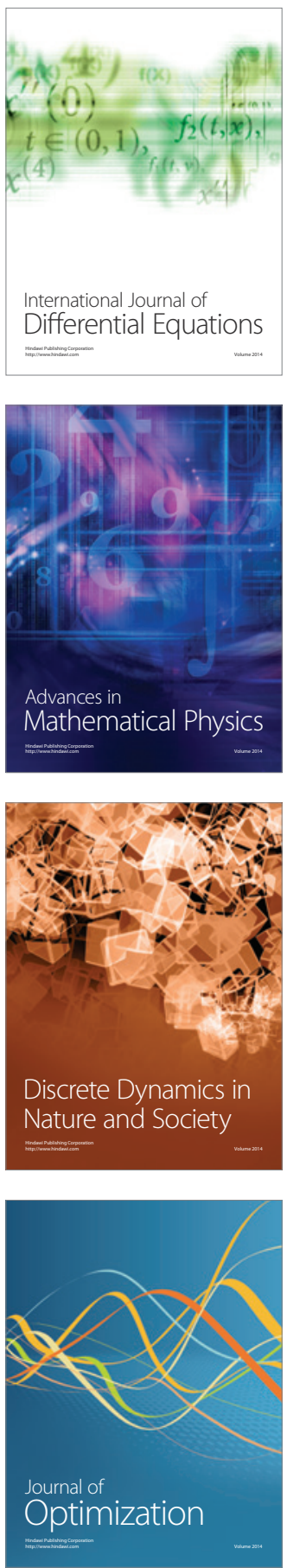